\title{
Summary Report for the Analysis of the Sludge Batch 6 (Macrobatch 7) DWPF Pour Stream Glass Sample for Canister S03472
}

F.C. Johnson

February 2012

Savannah River National Laboratory

Savannah River Nuclear Solutions, LLC Aiken, SC 29808

Prepared for the U.S. Department of Energy under contract number DE-AC09-08SR22470.

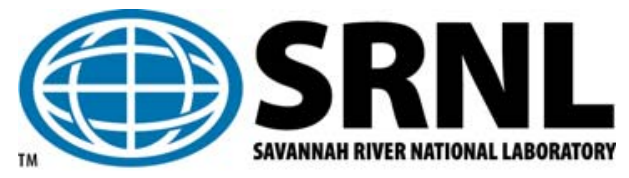


SRNL-STI-2012-00039

Revision 0

\section{DISCLAIMER}

This work was prepared under an agreement with and funded by the U.S. Government. Neither the U.S. Government or its employees, nor any of its contractors, subcontractors or their employees, makes any express or implied:

1. warranty or assumes any legal liability for the accuracy, completeness, or for the use or results of such use of any information, product, or process disclosed; or

2. representation that such use or results of such use would not infringe privately owned rights; or

3. endorsement or recommendation of any specifically identified commercial product, process, or service.

Any views and opinions of authors expressed in this work do not necessarily state or reflect those of the United States Government, or its contractors, or subcontractors.

Printed in the United States of America

Prepared for

U.S. Department of Energy 
Keywords: $D W P F$

Glass

Sludge Batch 6

Retention: Permanent

\title{
Summary Report for the Analysis of the Sludge Batch 6 (Macrobatch 7) DWPF Pour Stream Glass Sample for Canister S03472
}

\author{
F.C. Johnson
}

February 2012

Savannah River National Laboratory

Savannah River Nuclear Solutions, LLC

Aiken, SC 29808

Prepared for the U.S. Department of Energy under contract number DE-AC09-08SR22470.

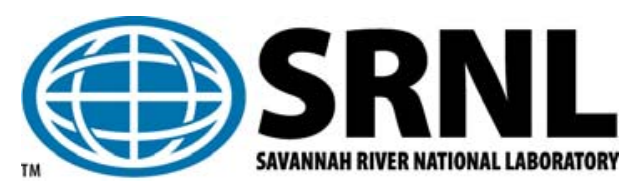




\section{REVIEWS AND APPROVALS}

\section{AUTHORS:}

F.C. Johnson, Process Technology Programs

Date

TECHNICAL REVIEW:

D.K. Peeler, Process Technology Programs

Date

APPROVAL:

C.C. Herman, Manager

Date

Process Technology Programs

Date

S.L. Marra, Manager

Environmental \& Chemical Process Technology Research Programs

J.E. Occhipinti, Manager

Date

Waste Solidification Engineering 


\subsection{Summary}

In order to comply with the Waste Acceptance Specifications in Sludge Batch 6 (Macrobatch 7), Savannah River National Laboratory personnel performed characterization analyses on the Defense Waste Processing Facility (DWPF) pour stream glass sample collected while filling canister S03472. This report summarizes results of the characterization, which indicate that the DWPF produced glass that is significantly more durable than the Environmental Assessment glass. Results and further details are documented in "Analysis of DWPF Sludge Batch 6 (Macrobatch 7) Pour Stream Glass Samples,” SRNL-STI-2011-00555.

Table 1. Measured Reportable Oxides ${ }^{\mathrm{a}}$

\begin{tabular}{|c|c|}
\hline Oxide & Concentration (wt \%) \\
\hline $\mathrm{Al}_{2} \mathrm{O}_{3}$ & 8.63 \\
\hline $\mathrm{B}_{2} \mathrm{O}_{3}$ & 4.55 \\
\hline $\mathrm{Fe}_{2} \mathrm{O}_{3}$ & 8.74 \\
\hline $\mathrm{Li}_{2} \mathrm{O}$ & 4.92 \\
\hline $\mathrm{MnO}$ & 2.19 \\
\hline $\mathrm{Na}_{2} \mathrm{O}$ & 14.86 \\
\hline $\mathrm{NiO}$ & 1.02 \\
\hline $\mathrm{SiO}_{2}$ & 49.10 \\
\hline $\mathrm{ThO}_{2}$ & 1.00 \\
\hline $\mathrm{U}_{3} \mathrm{O}_{8}$ & 1.83 \\
\hline
\end{tabular}

Table 2. Normalized PCT Results (g/L)

\begin{tabular}{|c|c|c|c|c|}
\hline Glass ID & NL B & NL Li & NL Na & NL Si \\
\hline EA $^{\text {b }}$ - Measured & 16.60 & 9.56 & 13.36 & 3.96 \\
\hline St. Dev. & 0.02 & 0.04 & 0 & 0 \\
\hline \% RSD & 0.1 & 0.4 & 0 & 0 \\
\hline EA $^{\text {c }}$ Published & 16.7 & 9.6 & 13.3 & 3.9 \\
\hline St. Dev. & 1.2 & 0.7 & 0.9 & 0.4 \\
\hline \% RSD & 7 & 7 & 7 & 10 \\
\hline SB6 & 0.69 & 0.81 & 0.85 & 0.49 \\
\hline St. Dev. & 0.02 & 0.01 & 0.02 & 0.01 \\
\hline \% RSD & 2.5 & 1.6 & 2.8 & 2.8 \\
\hline
\end{tabular}

${ }^{a}$ Greater than $0.5 \mathrm{wt} \%$ on an elemental basis. Note that only the aqua regia data was used for Ca, K, Na, S and Zr. Both the aqua regia and peroxide fusion data were used for all other elements.

${ }^{\mathrm{b}}$ Average of duplicate results.

${ }^{\text {c }}$ C.M. Jantzen, N.E. Bibler, D.C. Beam, C.L. Crawford, and M.A. Pickett, "Characterization of the Defense Waste Processing Facility (DWPF) Environmental Assessment (EA) Glass Standard Reference Material,” Westinghouse Savannah River Company, Aiken, SC, WSRC-TR-92-346, Rev. 1, 1993.

d Average of quadruplicate results. 
Table 3. Measured Reportable Radionuclides

\begin{tabular}{|c|c|}
\hline Radionuclide & $\begin{array}{c}\text { Concentration } \\
\text { (Ci/kg) }\end{array}$ \\
\hline Sr-90 & $5.9 \mathrm{E}+00$ \\
\hline Zr-93 & $5.3 \mathrm{E}-04$ \\
\hline Tc-99 & $<1.3 \mathrm{E}-04$ \\
\hline Cs-137 & $1.3 \mathrm{E}+00$ \\
\hline Th-232 & $9.3 \mathrm{E}-07$ \\
\hline $\mathrm{U}-233$ & $7.0 \mathrm{E}-05$ \\
\hline $\mathrm{U}-234$ & $4.2 \mathrm{E}-05$ \\
\hline $\mathrm{U}-235$ & $2.3 \mathrm{E}-07$ \\
\hline $\mathrm{U}-236$ & $6.3 \mathrm{E}-07$ \\
\hline $\mathrm{U}-238$ & $5.1 \mathrm{E}-06$ \\
\hline $\mathrm{Np}-237$ & $1.7 \mathrm{E}-05$ \\
\hline $\mathrm{Pu}-238$ & $1.4 \mathrm{E}-01$ \\
\hline $\mathrm{Pu}-239$ & $7.7 \mathrm{E}-03$ \\
\hline $\mathrm{Pu}-240$ & $2.9 \mathrm{E}-03$ \\
\hline $\mathrm{Pu}-241$ & $3.7 \mathrm{E}-02$ \\
\hline $\mathrm{Pu}-242$ & $<1.4 \mathrm{E}-05$ \\
\hline $\mathrm{Am}-241$ & $1.4 \mathrm{E}-02$ \\
\hline
\end{tabular}




\section{Distribution:}

J.W. Amoroso, 999-W

C. J. Bannochie, 773-42A

A. B. Barnes, 999-W

J. M. Bricker, 704-27S

M. A. Broome, 704-29S

C.L. Crawford, 773-42A

T.B. Edwards, 999-W

T. L. Fellinger, 704-26S

S. D. Fink, 773-A

K.M. Fox, 999-W

B. J. Giddings, 786-5A

J. M. Gillam, 766-H

C. C. Herman, 999-W

R. N. Hinds, 704-S

E. W. Holtzscheiter, 704-15S

J. F. Iaukea, 704-30S

P. R. Jackson, DOE-SR, 703-46A

C.M. Jantzen, 773-A

F.C. Johnson, 999-W

M. T. Keefer, 241-156H

D.P. Lambert, 999-W

S. L. Marra, 773-A

D.W. McIlmoyle, 766-H

J.D. Newell, 999-W

J. E. Occhipinti, 704-S

J.M. Pareizs, 773-A

D. K. Peeler, 999-W

F. M. Pennebaker, 773-42A

J. W. Ray, 704-S

A.R. Shafer, 704-27S

H. B. Shah, 766-H

D. C. Sherburne, 704-S

A. V. Staub, 704-27S

M. E. Stone, 999-W

K. H. Subramanian, 766-H

J. P. Vaughan, 773-41A

W. R. Wilmarth, 773-A

J.R. Zamecnik, 999-W 